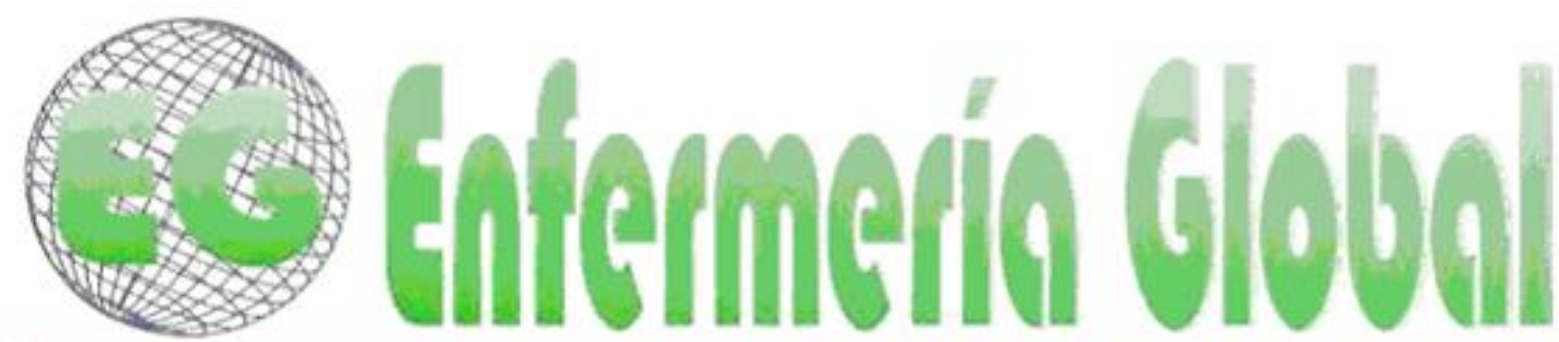

ISSN 1695-6141

$N^{\circ} 43$

Revista electrónica trimestral de Enfermería

Julio 2016

www.um.es/egloball

CLÍNICA

\title{
Características del paciente quemado usuario de opioide y factores que contribuyen a depresión respiratoria
}

Características do paciente queimado em uso de opióide e os fatores contribuintes para depressão respiratória

Characteristics of burned patient using opioid and contributory factors for respiratory depression

${ }^{*}$ Henrique, Danielle Mendonça **Silva, Lolita Dopico ${ }^{* * *}$ Pareira, Sandra Regina Maciqueira

\begin{abstract}
*Enfermera del Centro de Quemados del Hospital do Andarai.. E-mail: danimendh@gmail.com
**Profesora permanente del programa de posgraduación en Enfermería de la Universidad del estado de Rio de Janeiro ***Doctora en Enfermería , Profesora Adjunta del Departamento de Enfermería Médico-Quirúrgica de la FEUERJ. Brasil.
\end{abstract}

Palabras clave: Enfermería; Quemaduras; Analgésicos opióides; insuficiencia respiratoria

Palavras chave: Enfermagem; Queimaduras; Analgésicos Opióides; Insuficiência respiratória

Keywords: Nursing; Burned; Opioid; Respiratory depression.

\section{RESUMEN}

Objetivo: Describir las características de los pacientes quemados usuarios de opioides e identificar los factores que predisponen a depresión respiratoria.

Método: Transversal retrospectivo, en el que se analizaron 272 historiales de pacientes quemados internados de 2011 a 2013 en un Centro de Tratamiento de Quemados (CTQ). Se seleccionaron historiales completos de pacientes, con registro relleno de alta u óbito, periodo de internamiento mínimo de 48 horas, mayores de 18 años, usuarios de opioides por diferentes vías de administración, monitoreados por oximetría de pulso y sin empleo de prótesis de ventilación. Todos los episodios de depresión respiratoria se identificaron a través de cómo mínimo dos indicadores de entre los siguientes: la administración de Naloxone, registro de presión arterial inferior a $110 / 60 \mathrm{mmHg}$, entubamiento orotraqueal, oximetría de pulso inferior a $90 \%$, frecuencia respiratoria inferior a $10 \mathrm{irpm}$, necesidad de oferta de oxígeno e interrupción abrupta del opioide. .

Resultados: Este estudio identificó que el $28,58 \%$ de los pacientes quemados atendidos en una unidad de tratamiento de quemados presentaron depresión respiratoria. Predominaron en el grupo con depresión respiratoria pacientes jóvenes del sexo masculino, con mortalidad elevada, quemaduras de 2 ํㅡ y 3 grado fueron la mayoría, corroborando la mayoría de los estudios epidemiológicos acerca de las características de los pacientes quemados. Los factores predominantes para depresión respiratoria 
identificados en la población de pacientes quemados usuarios de opioides fueron insuficiencia renal aguda, etilismo, enfermedades cardiovasculares.

Conclusión: El enfermero debe monitorear a todos los pacientes usuarios de opioides en relación a los parámetros de ventilación y nivel de coincidencia para prevenir e identificar precozmente sedación excesiva y depresión respiratoria, especialmente los pacientes en quienes se identifiquen dos o más factores de predisposición para DR.

\section{RESUMO}

Objetivo: Descrever as características dos pacientes queimados em uso de opióide, e de identificar os fatores predisponentes para depressão respiratória.

Metodo: Transversal retrospectivo, em que foram analisados 272 prontuários de pacientes queimados internados entre os anos de 2011 a 2013 em um Centro de Tratamento de Queimados .Selecionados prontuários completos de pacientes, com registro preenchido da alta ou óbito, período de internação mínimo de 48 horas, maiores de 18 anos, que utilizaram opióides por diferentes vias de administração, monitorados com oximetria de pulso e sem emprego de prótese ventilatória. Todos os episódios de depressão respiratória foram identificados através de no mínimo dois indicadores dentre os seguintes: a administração de Naloxone. registro de pressão arterial menor de $110 / 60 \mathrm{mmHg}$, intubação orotraqueal, oximetria de pulso menor que 90\%, frequência respiratória menor que 10 irpm, necessidade da oferta de oxigênio e interrupção abrupta do opióide.

Resultados: Este estudo identificou que $28,58 \%$ dos pacientes queimados atendidos numa unidade de tratamento de queimados apresentaram depressão respiratória. Predominaram no grupo com depressão respiratória pacientes jovens do sexo masculino, com mortalidade elevada, queimaduras de $2^{0}$ e $3^{\circ}$ grau foram a maioria, corroborando com a maioria dos estudos epidemiológicos acerca das características dos pacientes queimados. Os fatores predominantes para depressão respiratória identificados na população de pacientes queimados em uso de opióide foram insuficiência renal aguda, etilismo, doenças cardiovasculares.

Conclusao: $O$ enfermeiro deve monitorar os pacientes em uso de opióide, em relação aos parâmetros ventilatórios e nível de consciência, para prevenir e identificar precocemente sedação excessiva e depressão respiratória, principalmente naqueles em que forem identificados dois ou mais fatores predisponentes para depressão respiratória.

\section{ABSTRACT}

Objective: This study aims to describe the characteristics of burned patients using opioid and to identify the predisposing factors for respiratory depression.

Method: For this study, a retrospective cross-sectional method was used, analyzing 272 medical records of burned patients hospitalized from 2011 to 2013 in a Burn Treatment Center. Complete records of patients over 18 years old were selected with completed registration of discharge or death that had remained in the hospital for a minimum of 48 hours and were under use of opioids by different administration routes and monitored with pulse oximetry and without using ventilatory prosthesis. All episodes of respiratory depression were identified by at least two indicators from the administering Naloxone.

Results: Based on this study, $28.58 \%$ of burned patients treated in a burn care unit with respiratory depression were found. In the group with respiratory depression, young male patients were predominant with high mortality, and the most patients had $2^{\text {nd }}$ and $3^{\text {rd }}$ degree burns, confirming the most epidemiological studies concerning the characteristics of burned patients. The predominant factors for respiratory depression identified in the population of burned patients using opioids were acute renal failure, alcoholism, and cardiovascular diseases.

Conclusion: Nurses should monitor all patients using opioids in relation to the ventilatory parameters and consciousness level to prevent and identify early excessive sedation and respiratory depression, particularly in patients with two or more predisposing factors for respiratory depression 


\section{INTRODUCCIÓN}

Quemaduras son lesiones tisulares de gran morbilidad y mortalidad que generan una importante carga financiera global. Datos recogidos de diversos centros hospitalarios y suministrados por la Sociedad Americana de Quemaduras revelan una estimativa de 500.000 casos de lesiones al año recibiendo tratamiento médico en los EE.UU., de los cuales 40.000 ingresos y mortalidad aproximada de 4.000 pacientes, en su mayoría víctimas de accidentes en domicilio. En Brasil, a pesar de la inexistencia de un efectivo sistema centralizador de informaciones, se estima que estas cifras sean aún mayores ${ }^{1}$.

Los analgésicos opioides son los más empleados para tratamiento del dolor de quemadura. Los factores que justifican la elección de esta clase de medicamentos incluyen analgesia potente; el perfil farmacocinético (facilidad de absorción, distribución y excreción de los medicamentos de esta clase); variedad de vías de administración y que aún pueden proporcionar un cierto grado de sedación según la dosis administrada, lo que puede ser ventajoso, particularmente durante los procedimientos de cuidados con la quemadura ${ }^{2}$.

Los opioides más empleados habitualmente en la práctica clínica en pacientes gran quemados son los agonistas morfínicos, que selectivamente se unen a los receptores mu $(\mu)$, pues son los que tienen efecto analgésico más potente, aunque son los más peligrosos, por el riesgo de causar depresión en el sistema nervioso central y como consecuencia, sedación excesiva y depresión respiratoria ${ }^{2}$.

Por su potencial de provocar sedación excesiva y depresión respiratoria, los analgésicos opioides intravenosos, transdérmicos de empleo oral (incluyendo líquidos concentrados y formulaciones "deliberación inmediata" o "prolongada") están en el rol de los Medicamentos Potencialmente Peligrosos (MPP), definidos así por ser medicamentos que presentan mayor riesgo de provocar daños en los pacientes cuando se produce un fallo en su proceso de empleo ${ }^{3}$.

Esta clase de analgésicos puede causar diversos efectos colaterales, tanto a corto como a largo plazo. De entre los efectos asociados al empleo del opioide, el más grave es la depresión respiratoria, que se considera como evento adverso, evento al que generalmente precede una sedación excesiva ${ }^{4}$.

En este sentido se sabe que hay factores que pueden contribuir a la aparición no intencional de sedación excesiva y depresión respiratoria, generalmente destacados por la literatura como edad avanzada, enfermedad pulmonar obstructiva crónica, apnea del sueño, etilismo, insuficiencia cardíaca congestiva, hipertensión arterial, diabetes melitus, insuficiencia renal aguda e insuficiencia hepática ${ }^{4-6}$.

Todos estos aspectos que implican el empleo de opioides fundamentaron la realización de una investigación con los objetivos de describir las características de los pacientes quemados en toma de opioides, y de identificar los factores que predisponen a depresión respiratoria en estos pacientes, según lo citado en la literatura. 


\section{MÉTODO}

Estudio transversal retrospectivo en el que se analizaron 272 historiales de pacientes quemados internados entre 2011 y 2013 en un Centro de Tratamiento de Quemados (CTQ) de un hospital público federal de gran tamaño, en el municipio de Rio de Janeiro. Se seleccionaron historiales completos de pacientes, con registro relleno de alta u óbito, periodo de ingreso mínimo de 48 horas, mayores de 18 años, que emplearon opioides por diferentes vías de administración, monitoreados con oximetría de pulso y sin empleo de prótesis de ventilación.

Todos los episodios de depresión respiratoria se identificaron a través de como mínimo dos de los indicadores de entre los siguientes: la administración de Naxolone, registro de presión arterial inferior a $110 / 60 \mathrm{mmHg}$, entubamiento orotraqueal, oximetría de pulso inferior a $90 \%$, frecuencia respiratoria inferior a 10 irpm, necesidad de oferta de oxígeno e interrupción abrupta del opioide.

Se analizaron las siguientes variables: sexo, destino, grado de quemadura, realización de cirugía de autoinjertos, días de ingreso, edad y superficie corporal quemada. Entre los factores de predisposición mencionados en la literatura se encontró en esta población de pacientes quemados, insuficiencia renal, la hipoalbuminea, la enfermedad cardiovascular, etilismo y tabaquismo.

Los historiales que atendieron los criterios de selección se dividieron en dos grupos. El grupo (G1) correspondió a los pacientes que presentaron DR y el grupo (G2) formado por pacientes en el que no se constató DR. Las variables se sometieron a estadística descriptiva e inferencial, se consideró un intervalo de confianza de $95 \%$ para cálculo del $p$ a través del test de Fisher. También se emplearon medidas de asociación como OddsRatio (OR) y Riesgo Relativo (RR).

El estudio se sometió a aprobación Institucional a través de carta de autorización para investigación y al comité de ética en investigación de la institución y autorizado por el parecer consustanciado del CEP - no 453.911/2013.

\section{RESULTADOS}

Se encontraron 42 historiales que atendieron a los criterios de selección de la investigación y de estos en un $28,58 \%(n=1)$ se identificaron casos de depresión respiratoria. Se produjo la pérdida de 28 historiales que eran de menores de 18 años, de otros 25 por ser pacientes en óbito antes de $48 \mathrm{~h}$ de ingreso y los restantes por tratarse de pacientes que no tuvieron oximetría de pulso monitoreada.

\section{Características de los pacientes quemados que emplean opioides.}

Se presentan en la tabla 1 las principales características de los pacientes quemados, que fueron distribuidos en dos grupos, el de los pacientes que tuvieron depresión respiratoria (D.R.), llamado G1 y el de los pacientes sin DR, denominado G2. 
Tabla 1 - Características de los pacientes quemados. Rio de Janeiro, 2011-2013.

\begin{tabular}{|c|c|c|c|c|c|c|c|}
\hline $\begin{array}{l}\text { Características } \\
\text { Clínicas }\end{array}$ & & $\begin{array}{l}\text { G1 } \\
\text { ntes con } \\
(n=12)\end{array}$ & & $\begin{array}{l}\text { G2 } \\
\text { entes sin } \\
(n=30)\end{array}$ & $p$ & & $\begin{array}{l}\text { Total } \\
n=42)\end{array}$ \\
\hline Sexo & $\mathrm{n}$ & $\%$ & $\mathrm{n}$ & $\%$ & & $\mathrm{~N}$ & $\%$ \\
\hline Masculino & 8 & $19,04 \%$ & 23 & $54,76 \%$ & 0.7632 & 31 & $73,80 \%$ \\
\hline Femenino & 4 & $9,52 \%$ & 7 & $16,66 \%$ & & 11 & $26,19 \%$ \\
\hline Alta & 4 & $9,52 \%$ & 24 & $57,14 \%$ & 0.01238 & 28 & $66,66 \%$ \\
\hline $\begin{array}{l}\text { Óbito } \\
\text { Grado de quemadura }\end{array}$ & 8 & $19,04 \%$ & 6 & $14,28 \%$ & & 14 & $33,33 \%$ \\
\hline $2^{\circ}$ & 4 & $9,52 \%$ & 17 & $40,47 \%$ & 0.3058 & 21 & $50 \%$ \\
\hline Cirugía de Auto-injerto & 8 & $19,04 \%$ & 13 & $30,95 \%$ & & 21 & $50 \%$ \\
\hline Sí & 2 & $4,76 \%$ & 13 & $30,95 \%$ & 0.1987 & 15 & $35,71 \%$ \\
\hline No & 10 & $23,80 \%$ & 17 & $40,47 \%$ & & 27 & $64,28 \%$ \\
\hline
\end{tabular}

$\mathrm{p}<0,05$ calculado por el Test exacto de Fisher (IC:95\%)

El sexo masculino fue predominante, tanto en el grupo con DR (19,04\%) como en la población en general $(73,80 \%)$. El óbito predominó en el grupo con DR. Las quemaduras de $2^{\circ}$ y $3^{\circ}$ grados, consideradas más graves, fueron predominantes en pacientes con depresión respiratoria. Sin embargo, entre los pacientes sin DR predominaron las quemaduras de $2^{\circ}$ grado.

Solo $4,76 \%$ de los pacientes con DR fueron sometidos a cirugía de auto-injerto. Este pequeño número de cirugías en este grupo se explica porque se realizan generalmente en la fase final del tratamiento del paciente quemado, y la mayoría de los pacientes con DR alcanzaron el óbito antes de llegar a esta fase.

La única variable que presentó diferencia significativa entre los grupos fue el destino, prevaleciendo el óbito en el grupo con DR ( $p=0,01238)$. La tabla 2 presenta las características de los pacientes quemados en las variables continuas.

Tabla 2 - Características de los pacientes quemados . Rio de Janeiro, 2011-2013.

\begin{tabular}{|c|c|c|c|c|c|c|}
\hline $\begin{array}{l}\text { Características } \\
\text { Clínicas }\end{array}$ & \multicolumn{2}{|c|}{ G1 } & \multicolumn{2}{|c|}{ G2 } & \multicolumn{2}{|c|}{$\begin{array}{l}\text { Total } \\
(\mathrm{n}=42)\end{array}$} \\
\hline & $\begin{array}{l}\text { Media } \\
(+\quad \text { DP })\end{array}$ & Mediana & $\begin{array}{l}\text { Media } \\
(+\quad \text { DP })\end{array}$ & Mediana & $\begin{array}{l}\text { Media } \\
(+\quad \text { DP })\end{array}$ & Mediana \\
\hline Días de ingreso & $\begin{array}{c}34,00 \\
(17,20)\end{array}$ & 17 & $\begin{array}{l}34,27 \\
(17,2)\end{array}$ & 31,5 & $\begin{array}{c}34,19 \\
(19.97)\end{array}$ & 25,5 \\
\hline Edad (años) & $\begin{array}{c}47,58 \\
(17,75)\end{array}$ & 47,5 & $\begin{array}{l}39,16 \\
(12,7)\end{array}$ & 34,5 & $\begin{array}{c}42,00 \\
(14,00)\end{array}$ & 39 \\
\hline
\end{tabular}




\begin{tabular}{|l|llllll|}
\hline Superficie & 50,17 & 50 & $\begin{array}{c}34,93 \\
(16,4)\end{array}$ & 36 & $\begin{array}{c}39,29 \\
(15,60)\end{array}$ & 39,25 \\
\hline Corporal & $(8,53)$ & & & \\
\hline
\end{tabular}

$\mathrm{p}<0,05$ calculado por el Test exacto de Fisher (IC:95\%)

Respecto al tiempo de ingreso, el grupo que presentó DR, presentó una mediana menor, lo que puede explicarse por la alta mortalidad en esta población. En relación a la edad, los pacientes con DR presentaron edad con mediana de 47,5 años. La superficie corporal quemada (SCQ) de los pacientes con DR fue mayor (mediana de $50 \%$ ) siendo un indicativo de gravedad en este grupo.

\section{Factores de predisposición para depresión respiratoria}

No se encontró en la población estudiada, pacientes con DPOC, apnea de f, insuficiencia hepática, ICC y diabetes. La tabla 3 presenta distribución de los factores de predisposición para depresión respiratoria, constatándose que no hubo significancia estadística entre los dos grupos. La insuficiencia renal e hipoalbuminemia fueron los factores de predisposición que predominaron en el grupo con DR.

Tabla 3 - Distribución de los factores de predisposición al DR. Rio de Janeiro, 2011-2013

\begin{tabular}{|c|c|c|c|c|c|c|c|}
\hline \multirow[t]{2}{*}{$\begin{array}{l}\text { Factores de } \\
\text { Predisposición al } \\
\text { DR }\end{array}$} & \multicolumn{2}{|c|}{$\begin{array}{c}\text { G1 } \\
\text { Pacientes con } \\
\text { DR }(n=12)\end{array}$} & \multicolumn{2}{|c|}{$\begin{array}{c}\text { G2 } \\
\text { Pacientes sin } \\
\text { DR }(n=30)\end{array}$} & \multirow[t]{2}{*}{$p$} & \multicolumn{2}{|c|}{$\begin{array}{c}\text { Total } \\
(n=42)\end{array}$} \\
\hline & $\mathrm{n}$ & $\%$ & $n$ & $\%$ & & $\mathrm{~N}$ & $\%$ \\
\hline Insuficiencia Renal & & & & & & & \\
\hline Sí & 11 & $\begin{array}{c}26,19 \\
\%\end{array}$ & 19 & $\begin{array}{c}45,23 \\
\%\end{array}$ & 0.1342 & 30 & $\begin{array}{c}71,42 \\
\%\end{array}$ \\
\hline No & 1 & $2,38 \%$ & 11 & $\begin{array}{c}26,19 \\
\%\end{array}$ & & 12 & $\begin{array}{c}28,57 \\
\%\end{array}$ \\
\hline Etilismo & & & & & & & \\
\hline Sí & 3 & $7,14 \%$ & 4 & $9,52 \%$ & 0.7788 & 7 & $\begin{array}{c}16,66 \\
\%\end{array}$ \\
\hline No & 9 & $\begin{array}{c}21,42 \\
\%\end{array}$ & 26 & $\begin{array}{c}61,90 \\
\%\end{array}$ & & 35 & $\begin{array}{c}83,22 \\
\%\end{array}$ \\
\hline $\begin{array}{l}\text { Enfermedad } \\
\text { Cardiovascular }\end{array}$ & & & & & & & \\
\hline 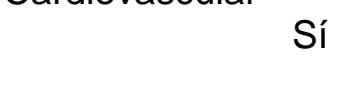 & 6 & $\begin{array}{c}14,28 \\
\%\end{array}$ & 7 & $\begin{array}{c}16,66 \\
\%\end{array}$ & 0.1903 & 13 & $\begin{array}{c}30,95 \\
\%\end{array}$ \\
\hline No & 6 & $\begin{array}{c}14,28 \\
\%\end{array}$ & 23 & $\begin{array}{c}54,76 \\
\%\end{array}$ & & 29 & $\begin{array}{c}69,04 \\
\%\end{array}$ \\
\hline Hipoalbuminemia & & & & & & & \\
\hline Sí & 11 & $\begin{array}{c}26,19 \\
\%\end{array}$ & 28 & $\begin{array}{c}66,66 \\
\%\end{array}$ & $\begin{array}{c}>0.999 \\
9\end{array}$ & 39 & $\begin{array}{c}92,85 \\
\%\end{array}$ \\
\hline No & 1 & $2,38 \%$ & 2 & $4,76 \%$ & & 3 & $7,14 \%$ \\
\hline Tabaquismo & 4 & $9,52 \%$ & 15 & $\begin{array}{c}35,71 \\
\%\end{array}$ & 0.5272 & 19 & $\begin{array}{c}45,23 \\
\%\end{array}$ \\
\hline No & 8 & $\begin{array}{c}19,04 \\
\%\end{array}$ & 15 & $\begin{array}{c}35,71 \\
\%\end{array}$ & & 23 & $\begin{array}{c}54,76 \\
\%\end{array}$ \\
\hline
\end{tabular}

$\mathrm{p}<0,05$ calculado por el test exacto de Fisher (IC:95\%). 
A pesar de no haberse constatado diferencias significativas entre los grupos en relación a los factores de predisposición a DR, se realizó un cálculo de asociación entre estos factores y la presencia o no de DR. En el cuadro 1 se presentan las medidas de asociación entre Depresión respiratoria y factores de predisposición a este evento.

Cuadro 1 - Asociación entre Depresión Respiratoria y factores de predisposición. RJ. 2011-2013.

\begin{tabular}{|c|c|c|c|c|c|}
\hline $\begin{array}{c}\text { Factores de } \\
\text { Predisposición }\end{array}$ & $p$ & $\begin{array}{l}\text { OR } \\
\text { (IC) }\end{array}$ & $\mathrm{RR}$ & $\begin{array}{c}\text { Razón } \\
\text { Expuestos }\end{array}$ & $\begin{array}{l}\text { Razón no } \\
\text { Expuestos }\end{array}$ \\
\hline $\begin{array}{c}\text { Insuficiencia } \\
\text { Renal }\end{array}$ & 0.1342 & $\begin{array}{c}6.368 \\
(0.7217-56.2)\end{array}$ & 4,4 & $36.67 \%$ & $8.333 \%$ \\
\hline Etilismo & 0.6236 & $\begin{array}{c}2.167 \\
(0.4047- \\
11.6)\end{array}$ & 1,6 & $42.86 \%$ & $25.71 \%$ \\
\hline $\begin{array}{c}\text { Enfermedad } \\
\text { Cardiovascular }\end{array}$ & 0.1903 & $\begin{array}{c}3.286 \\
(0.7999- \\
13.5)\end{array}$ & 2,2 & $46.15 \%$ & $20.69 \%$ \\
\hline Hipoalbuminemia & $>0.999999$ & $\begin{array}{c}0.7857 \\
(0.06452-9.5)\end{array}$ & 0.8462 & ------ & ------- \\
\hline Tabaquismo & 0.5272 & $\begin{array}{c}0.5 \\
(0.1236- \\
2.02)\end{array}$ & 0.6053 & ------------- & ----------- \\
\hline
\end{tabular}

Se constató la asociación positiva entre DR e insuficiencia renal, etilismo y enfermedad cardiovascular.

La insuficiencia aguda, presentó la mayor asociación, constatándose que el riesgo de DR en pacientes con insuficiencia renal aguda es 4,4 veces mayor que la del paciente sin este factor de predisposición. La posibilidad de que estos pacientes presenten DR es 6,3 veces mayor que los que no presenten insuficiencia renal.

El riesgo de DR en un paciente alcoholizado excede 1,6 veces la de un paciente sobrio. Siendo la posibilidad de que los alcoholizados presenten este desenlace 2,1 veces mayor que los que no son consumidores de bebidas alcohólicas.

La presencia de enfermedad cardiovascular presentó asociación positiva, siendo el riesgo de que pacientes con enfermedades cardiovasculares presenten DR 2,2 veces mayor. La posibilidad de que los cardiópatas presenten DR excede 3,2 al número de pacientes no cardiópatas.

La hipoalbuminemia se consideró una variable que confunde, pues se trata de una condición del paciente quemado, lo que explica que casi todos los pacientes estudiados presenten hipoalbuminemia. Respecto al tabaquismo, no hubo asociación positiva.

\section{DISCUSIÓN}

\section{Características de los pacientes quemados usuarios de opioides}

Se encontró una tasa de $28,58 \%$ de DR, con predominio de hombres, con superficie corporal quemada extensa y profundidad de $2^{\circ}$ y $3^{\circ}$ que se configuran en indicadores 
de gravedad, y justificando así la mayor mortalidad en este grupo, que permanece una media de 17 días internado y con mediana de edad más elevada.

En este estudio, el sexo masculino fue predominante tanto en el grupo con y sin DR, dato compatible con otros estudios nacionales e internacionales en lo que se refiere a la prevalencia de las quemaduras entre individuos del sexo masculino ${ }^{7-9}$.

Los hombres todavía trabajan en mayor número de servicios en los que se exponen a actividades de mayor riesgo de accidentes, como manipulación de equipos mecánicos o trabajo en red de electricidad, manipulación de sustancias químicas, además de los combustibles, entre otros riesgos graves de accidentes ${ }^{9}$.

Respecto a la edad, el tramo de edad de pacientes adultos jóvenes coincide con la mayoría de estudios epidemiológicos de la población de quemados ${ }^{10-12}$. El predominio de casos de quemadura en jóvenes adultos de sexo masculino encontrado en esta investigación coincide con lo relatado en otros estudios epidemiológicos que relacionan este alto índice con individuos económicamente activos, expuestos a situaciones de riesgo en el ambiente de trabajo y por considerarse menos cautelosos que las mujeres. En este tramo de edad se concentra la mayor fuerza productiva de la población $^{13-15}$.

Respecto al destino de los pacientes, el óbito prevaleció en los pacientes con DR. Sin embargo, en los pacientes que no presentaron esta complicación, la mayoría tuvo el alta hospitalaria, lo que contradice las publicaciones actuales ${ }^{16}$. En los años noventa, entre un 50 y un $80 \%$ de los pacientes con quemaduras que suponen más el $50 \%$ de la superficie corporal fallecían debido a complicaciones como sepsis, choque e inviabilidad de múltiples órganos. Actualmente, la mayoría de estos pacientes presentan una evolución satisfactoria.

En lo que respecta a la profundidad de la quemadura, algunos estudios evidencian la prevalencia del segundo grado ${ }^{17,18}$, aunque se observa una variación de acuerdo con el perfil de la atención, unidades de quemados de gran tamaño detentan una estructura para recibir pacientes de mayor gravedad. En este estudio el grupo con DR era el de mayor gravedad, con quemaduras de $2^{\circ}$ y $3^{\circ}$ grado.

En lo referente al tiempo de ingreso en días, un estudio presentó una media menor ${ }^{19}$, mientras otros presentaron una tasa cercana a la mediana del grupo de pacientes en $\mathrm{DR}^{20,21}$. Adecuados recursos humanos y tecnológicos para el tratamiento de pacientes con lesión por quemadura provocan una tendencia a reducir el tiempo de ingreso y la tasa de mortalidad de los mismos. La eficiencia de los centros especializados en la atención de quemaduras, con la utilización rígida de protocolos técnicos basados en evidencias, con la realización de actos operatorios de forma precoz, un apoyo nutricional agresivo y técnicas de sustitución de piel reducen la morbilidad y mortalidad y, por tanto, el tiempo de internamiento ${ }^{8}$.

\section{Factores de predisposición para depresión respiratoria por opioides}

Respecto a los factores de predisposición a DR, se apreciaron: insuficiencia renal (complicación de la propia quemadura), enfermedad cardiovascular y por último etilismo. Todos estos factores, a pesar de no presentar diferencia estadística significativa, presentaron posibilidad de riesgo significativo. 
Estudios afirman que los factores de predisposición para depresión respiratoria pueden ser la edad superior a 65 años, enfermedad pulmonar obstructiva crónica, apnea del sueño, insuficiencia cardíaca congestiva, insuficiencia renal, hepática y además otros factores como dosis, vía de administración u horario de administración del opioide ${ }^{22,23}$. En este estudio se identificaron asociaciones positivas entre el acaecimiento de DR y la insuficiencia renal, etilismo y enfermedad cardiovascular.

En esta investigación los pacientes con insuficiencia renal aguda presentaron 6,3\% de posibilidad de DR, muy superior cuando se compara con un estudio que evidenció que los pacientes con niveles de urea elevados (> $30 \mathrm{mg} / \mathrm{dL}$ ) tenían 2,09 veces más posibilidades de desarrollar depresión respiratoria. El seguimiento de los niveles de urea, creatinina y encimas hepáticas de estos pacientes es fundamental para el enfermero, en la identificación de los pacientes de mayor riesgo, para que los mismos puedan ser señalizados y mejor monitoreados ${ }^{24}$. En el paciente quemado debido al incremento de la permeabilidad vascular, el organismo pierde cerca de $4,4 \mathrm{ml} / \mathrm{hg} / \mathrm{h}$, requiriendo por lo tanto una reposición volémica muy agresiva. En ausencia de una reposición adecuada, se producirá una reducción del débito cardíaco y del flujo plasmático renal, por lo que la insuficiencia renal es muy frecuente en este paciente. Constituye así un factor de predisposición importante para la depresión respiratoria, por la dificultad de eliminación por vía renal del opioide ${ }^{24-25}$.

La insuficiencia renal con posible significancia farmacológica se define por el Foodand Drug Administratio (FDA) como estimativa de clearence de creatinina inferior a $80 \mathrm{~mL} / \mathrm{min} / \mathrm{m} 2$. La insuficiencia renal moderada y grave están presentes cuando clearence de creatinina es de $30-50 \mathrm{~mL} / \mathrm{min} / \mathrm{m} 2$ e inferior a $30 \mathrm{~mL} / \mathrm{min} / \mathrm{m} 2$ respectivamente. Se recomienda que en estos pacientes, se inicie terapéutica con opioide en dosis reducidas, titular lentamente y monitorear las concentraciones del medicamento y eventos adversos ${ }^{26}$. Ante estas evidencias en el cuidado de pacientes quemados usuarios de opioides, el enfermero debe monitorear la hidratación venosa agresiva, el débito urinario, la función renal, así como instalar balance hídrico riguroso.

En este estudio, pacientes con enfermedades cardiovasculares evidenciaron una posibilidad de 3,2 y RR de 2,2 de llegar a presentar DR.

En lo relativo a enfermedades cardiovasculares, estudios comprueban que insuficiencia cardíaca congestiva, arritmia cardíaca, enfermedad de la arteria coronaria e hipertensión detentan una asociación significativa con el inicio súbito de eventos respiratorios críticos, con riesgo de muerte durante la analgesia del opioide ${ }^{23}$. Se recomienda que la función cardiovascular se monitoree durante la terapéutica con opioide, más específicamente al inicio del tratamiento y tras el incremento de la dosis. Pacientes internados deben ser monitoreados para señales de alteración cardiovascular (cianosis, frecuencia respiratoria reducida, reducción de frecuencia cardíaca, hipotensión, falta de aire, estertores y edema periférico). La hipotensión generalmente antecede a la depresión respiratoria y es por lo tanto, una señal importante ${ }^{26}$.

Dada la dificultad de prever el riesgo de efectos cardiovasculares, los opioides deben emplearse con extrema cautela en pacientes con este factor de predisposición. Entre los opioides de amplia acción, la metadona debe evitarse así como la morfina, oxicodona y oximorfina deben administrarse con precaución. Se ha recomendado 
Tramadol en pacientes con factores de riesgo cardiovascular elevado y puede ser una opción segura para pacientes para los que se indique un opioide menos fuerte ${ }^{27}$.

Respecto al etilismo, los pacientes presentaron 2,1 de posibilidades 1.6 de riesgo relativo de DR y se sabe que pacientes con historial de abuso de alcohol o drogas sufren mayor riesgo de dependencia cuando emplean opioides, lo que puede acarrear serio riesgo debido a la elevación gradual de las dosis en estos pacientes, produciéndose eventos adversos como depresión respiratoria ${ }^{28}$.

En el paciente alcohólico el mecanismo compensatorio del alcohol implica endorfina y los opioides pueden tener efectos psicológicos similares al efecto del alcohol, siendo necesarias dosis elevadas para alcanzar analgesia en estos pacientes, lo que puede ocasionar un riesgo, por la aparición de eventos adversos ${ }^{28}$.

El enfermero debe considerar el historial del paciente respecto al etilismo, y evaluar señales de dependencia, e incremento progresivo de dosis de opioides, clasificando al paciente como un riesgo potencial y manteniendo monitoreo para sedación excesiva y depresión respiratoria, con el fin de detectar precozmente cualquier complicación o intercurrencia.

Estos aspectos que implican el empleo de opioides fundamentan la importancia de la evaluación de la sedación durante la terapia con opioide, por enfermeros, ya que la sedación excesiva puede ser un aviso para evitar la depresión respiratoria. En este sentido, los enfermeros deben adoptar como primera práctica en el cuidado diario la evaluación de la sedación (consecuencia del empleo del opioide), y que se trata de una fase anterior al de la depresión respiratoria. Esta evaluación se realiza en escalas. En la práctica clínica, las escalas más empleadas para evaluar sedación son la escala de Ramsay (RSS), Richmond (RASS), y Riker (SAS). Sin embargo estudios $^{7,8}$ consideran la escala POSS la más indicada para evaluación de sedación en el paciente usuario de opioide, ya que la identificación de los niveles de sedación es más exacta. Se trata de una escala desarrollada por enfermeros que evalúa el nivel de conciencia del paciente, clasificándolo en despierto y alerta, ligeramente adormecido pero con fácil despertar, frecuentemente somnoliento aunque despierto, y somnoliento estando mínimamente responsivo a las respuestas verbales o estímulos físicos. Se sugieren intervenciones ante los dos últimos ítems que se consideran inaceptables y que exigen intervenciones a través del cuidado de enfermería. Las intervenciones serían el mantenimiento de la evaluación de monitoreo de parámetros de ventilación, de los niveles de sedación, reducción de la dosis de opioide de 25 a $50 \%$ y suspensión inmediata del opioide.

\section{CONCLUSIÓN}

Este estudio identificó que el $28,58 \%$ de los pacientes quemados atendidos en una unidad de tratamiento de quemados presentaron depresión respiratoria. Predominaron en el grupo con depresión respiratoria pacientes jóvenes del sexo masculino, con mortalidad elevada, quemaduras de $2^{\circ}$ y 3 grado, que fueron la mayoría, corroborando así la mayor parte de los estudios epidemiológicos que abordan los pacientes quemados.

Los factores predominantes para depresión respiratoria identificados en la población de pacientes quemados en toma de opioides, fueron insuficiencia renal aguda, etilismo y enfermedades cardiovasculares. 
El enfermero debe saber identificar al paciente que presenta riesgo para desarrollar la depresión respiratoria, y evaluarlo según la situación de riesgo, implementando medidas específicas como en pacientes renales, el monitoreo del débito urinario, función renal, balance hídrico, en los pacientes cardiópatas el monitoreo electrocardiográfico, además de señales de insuficiencia cardíaca.

El enfermero debe monitorear a todos los pacientes usuarios de opioides, en relación a los parámetros de ventilación y el nivel de conciencia, para prevenir e identificar precozmente sedación excesiva y depresión respiratoria, sobre todo en los pacientes en los que se identifiquen dos o más factores de predisposición a DR.

El conocimiento del monitoreo adecuado de los parámetros respiratorios, la aplicación de escala de sedación específica para pacientes que emplean opioides, y la implementación de protocolos que identifiquen factores que predisponen a la depresión respiratoria en los pacientes que emplean opioides, son herramientas esenciales para que el enfermero identifique precozmente este grave evento adverso.

Se espera que este estudio pueda contribuir para la producción brasileña acerca de la temática de seguridad medicamentosa, fomentando nuevos estudios en esta área, generando conocimiento y fuente de datos para consulta de los profesionales de sanidad, ya que la depresión respiratoria derivada del empleo de opioides, es un evento adverso extremadamente grave, que debe ser foco de discusión de todo el equipo multidisciplinario, generando procesos asistenciales seguros.

\section{REFERENCIAS}

1. Gimenes,G.A., Alferes F.C.B.A., Dorsa P., Barros, A.C.P, Gonella,H.A. Estudo epidemiológico de pacientes internados no Centro de Tratamento de Queimados do Conjunto Hospitalar de Sorocaba. Rev Bras Queimaduras. 2009 2015);8(1):14-7. [Acesso 12/01/2015]. Disponível em: http://revistas.um.es/eglobal/article/view/eglobal.14.1.181431/168611

2. Goodman \& Gilman's. The Pharmacological Basis of Therapeutics. Section I, Drugs Ac- ting on the Central Nervous System. 12ed. 2011.

3. Institute for Safe Medication Practices. ISMP's list of high-alert medications. Huntingdon Valley (PA): ISMP; 2012. [Acesso 12/01/2015]. Disponível em: http://www.ismp-brasil.org/site/index/medicamentos/

4. Jarzyna D et al. American Society for Pain Management Nursing Guidelines on Monitoring for Opioid- Induced Sedation and Respiratory Depression. Pain Management Nursing, 2011. V.12(3) p. 118-145. [Acesso 12/01/2015]. Disponível em: http://www.aspmn.org/documents/GuidelinesonMonitoringforOpioid-

InducedSedationandRespiratoryDepression.pdf

5. The Joint Commission. Safe use of opioids in hospitals. Sentinel Event Alert, Issue; 2012. [Acesso 12/01/2015]. Disponível em: http://www.jointcommission.org/assets/1/18/SEA 49 opioids 8212 final.pdf

6.Henrique D.M, Silva, L.D. O uso seguro de opioides em pacientes queimados: fundamentando o cuidado de enfermagem. Rev Bras Queimaduras. 2014;13(1):6-10. [Acesso 12/01/2015]. Disponível em: http://www.rbqueimaduras.com.br/detalhe artigo.asp?id=190

7. Forjuoh S.N., Burns in low- and middle-income countries: A review of available literature on descriptive epidemiology, risk factors, treatment, and prevention. Burns. 2006; 32: 529-537. [Acesso 12/01/2015]. Disponível em: http://www.ncbi.nlm.nih.gov/pmc/articles/PMC3188147/ 
8. Souza AA, Mattar CA, Almeida PCC, Faiwichow L, Fernandes FS, Neto ECA, et al. Perfil epidemiológico dos pacientes internados na Unidade de Queimaduras do Hospital do Servidor Público Estadual de São Paulo. Rev Bras Queimaduras. 2009;8(3):87-90. [Acesso 12/01/2015]. Disponível em: http://www.rbqueimaduras.com.br/detalhe artigo.asp?id=18

9.Lacerda L.A, Carneiro A.C., Oliveira A.F., Gragnani A., Ferreira L.M. Estudo epidemiológico da Unidade de Tratamento de Queimaduras da Universidade Federal de São Paulo. Rev Bras Queimaduras. 2010;9(3):82-8. [Acesso 12/01/2015]. Disponível em: http://www.rbqueimaduras.com.br/detalhe artigo.asp?id=40

10. Macedo JLS, Rosa SC. Estudo epidemiológico dos pacientes internados na Unidade de Queimados: Hospital Regional da Asa Norte, 1992- 1997. Brasília Med. 2000;37:87-92. [Acesso 12/01/2015]. Disponível em: http://www.scielo.br/scielo.php?script=sci nlinks\&ref=000114\&pid=S03650596200500010000300012\&lng=en

11. Onarheim $\mathrm{H}$, Jensen SA, Rosenberg BE, Guttormsen AB. The epidemiology of patients with burn injuries admitted to Norwegian hospitals in 2007. Burns. 2009;35(8):1142-6. [Acesso 12/01/2015]. Disponível em: http://www.ncbi.nlm.nih.gov/pubmed/19748742

12. Cruz B.F, CordovilP.B.L, Batista K.N.M. Perfil epidemiológico de pacientes que sofreram queimaduras no Brasil: revisão de literatura. Rev Bras Queimaduras. 2012;11(4):246-50. [Acesso 12/01/2015]. Disponível em: http://www.rbqueimaduras.com.br/detalhe artigo.asp?id=130

13. Montes SF, Barbosa MH, Sousa Neto AL. Aspectos clínicos e epidemiológicos de pacientes queimados internados em um Hospital de Ensino. Rev Esc Enferm USP. 2011;45(2):369-73. [Acesso 12/01/2015]. Disponível em: http://www.revistas.usp.br/reeusp/article/viewFile/40710/43983

14. Narikawa R, Michelski DA, Hiraki PY, Ueda T, Nakamoto HA, Tuma Jr P, et al. Análise epidemiológica da mão queimada no Hospital das Clínicas da Faculdade de Medicina da Universidade de São Paulo. Rev Bras Queimaduras. 2011;10(3):89-92. [Acesso 12/01/2015]. Disponível em: http://www.rbqueimaduras.com.br/detalhe artigo.asp?id=73

15 . Moreira CA, Reis IF, Costa ACSM. Perfil epidemiológico de pacientes atendidos na unidade de tratamento de queimados no município de Aracaju (SE). 2011; 10(4): 114-118. [Acesso 12/01/2015]. Disponível em: http://www.rbqueimaduras.com.br/detalhe artigo.asp?id=80

16. Bervian F, Maino MM, Schmidt MK, Silva VBG, Arnt R, Martins PDE. Estudo de mortalidade em pacientes tratados na unidade de queimados no hospital de pronto socorro de Porto Alegre. ACM Arq Catarin Med. 2007;36(supl 1):173-4. [Acesso 12/01/2015]. Disponível em: http://www.acm.org.br/revista/pdf/artigos/468.pdf

17. Pereira Júnior S, Bins Ely J, Sakae TM, Nolla A, Mendes FD. Estudo de pacientes vítimas de queimaduras internados no Hospital Nossa Senhora da Conceição em Tubarão - SC. ACM Arq Catarin Med. 2007;36(2):22-7. [Acesso 12/01/2015]. Disponível em: http://www.acm.org.br/revista/pdf/artigos/487.pdf

18. Reis I.F., Moreira C.A., Costa A.C.S.M. Estudo epidemiológico de pacientes internados na unidade de tratamento de queimados do hospital de urgência de Sergipe. Rev Bras Queimaduras. 2011;10(4):114-8. [Acesso 12/01/2015]. Disponível em: http://www.rbqueimaduras.org.br/detalhe artigo.asp?id=80

19. Miranda RE, Pacannaro RC, Pinheiro LF, Calil JA, Gragnani A, Ferreira LM. Trauma elétrico: análise de 5 anos. Rev Bras Queimaduras.2009;8(2):65-9. [Acesso 12/01/2015].

Disponível

em:

http://www.rbqueimaduras.org.br/detalhe artigo.asp?id=13 
20. Rimdeika R, Kazanavicius M, Kubilius D. Epidemiology of burns in Lithuania during 1991-2004. Medicina (Kaunas). 2008;44(7):541-7. [Acesso 12/01/2015]. Disponível em: http://www.ncbi.nlm.nih.gov/pubmed/18695351

21. Burton KR, Sharma VK, Harrop R, Lindsay R. A population-based study of the epidemiology of acute adult burn injuries in the Calgary Health Region and factors associated with mortality and hospital length of stay from 1995 to 2004 . Burns. 2009;35(4):572-9. [Acesso 12/01/2015]. Disponível em: http://www.ncbi.nlm.nih.gov/pubmed/19203840

22. Jungquist, Carla R; Karan, Suzanne; Perlis, Michael L. Risk Factors for OpioidInduced Excessive Respiratory Depression. Pain Management Nursing. 2011; 12 (3):180-187. [Acesso 12/01/2015]. Disponível em: http://www.medscape.com/viewarticle/749755 3

23.Ramachandran, Satya Krishna; Haider, Naeem; Saran, Kelly A.; Mathis, Michael; Kim, Joyce; Morris, Michelle; O'Reilly, Michael. Life-threatening critical respiratory events: a retrospective study of postoperative patients found unresponsive during analgesic therapy. Journal of Clinical Anesthesia. 2011; 23:207-213. [Acesso $12 / 01 / 2015]$.

Disponível

em: http://experts.umich.edu/pubDetail.asp?n=Satya+Krishna+Ramachandran\&u id=4252 \&oe $i d=1 \& 0$ id $=24 \& i d=79956047797$

24. Johnson, Robert G.; Arozullah, Ahsan M.; Neumayer, Leigh; Henderson, William G.; Hosokawa, Patrick; Khuri, Shukri F. Multivariable predictors of postoperative respiratory failure after general and vascular surgery: Results from the patient safety in surgery study. Journal of the American College of Surgeons. 2007; 204(6):1188-1198. [Acesso 12/01/2015]. Disponível em: http://www.journalacs.org/article/S10727515(07)00325-0/abstract

25. Gregoretti C, et al. Analgo-sedation of patients with burns outside the operating room. Drugs. 2008;68(17):2427-43. [Acesso 12/01/2015]. Disponível em: http://www.unboundmedicine.com/harrietlane/ub/citation/19016572/Analgo sedation of patients with burns outside the operating room

26. Smith H, Bruckenthal P. Implications of Opioid Analgesia for Medically Complicated Patients. Drugs Aging 2010; 27 (5): 417-433 [Acesso 12/01/2015]. Disponível em: http://www.ncbi.nlm.nih.gov/pubmed/20450239

27. Mason JW, Schwertschlag US, Klutzaritz V, Canafax DM. Electrocardiographic and cardiovascular diagnostic characteristics of patients receiving long-term opioid therapy for pain. J Opioid Manag. 2014 Mar-Apr;10(2):103-9. [Acesso 12/01/2015]. Disponível em: http://www.ncbi.nlm.nih.gov/pubmed/24715665

28. Elsayem A, Bruera E. Methadone-Induced Respiratory Depression in a Patient with a History of Alcoholism. Journal of palliative medicine. 2005; 8(5):1062-1066. [Acesso 12/01/2015]. Disponível em: http://www.ncbi.nlm.nih.gov/pubmed/?term=MethadoneInduced+Respiratory+Depression+in+a+Patient+with+a+History+of+Alcoholism

Recibido: 13 de Enero 2015; Aceptado: 10 de Febrero 2015

ISSN 1695-6141

(C) COPYRIGHT Servicio de Publicaciones - Universidad de Murcia 